\title{
The effect of stochastically environmental variability on transmission dynamics of echinococcosis
}

\author{
Lei Wang ${ }^{1}$, Kai Wang ${ }^{1}$, Yu Zhao ${ }^{2}$, and Daqing Jiang ${ }^{3}$ \\ ${ }^{1}$ Xinjiang Medical University \\ ${ }^{2}$ Ningxia Medical University \\ ${ }^{3}$ College of Science, China University of Petroleum(East China)
}

July 17, 2020

\begin{abstract}
Echinococcosis, one of the most serious zoonotic diseases, has a severe impact on the human health and economic development. This paper mainly investigates the effect of stochastically environmental variability on transmission dynamics of echinococcosis. Firstly, sufficient conditions of the extinction in the mean for the disease are obtained. In addition, by constructing a suitable stochastic Lyapunov function, the existence of the unique ergodic stationary distribution is established. Lastly, numerical simulations have been performed to not only verify our analytical results but also display that noise intensities would affect the dynamical behaviors of this model, (i) these noise intensities for three subgroups all have significantly negative impact on the extinction time for $\$ I_{-} H(t) \$$, in particular, when the noise intensity for the livestock $\$ \backslash$ sigma_L $\$$ increases, the extinction time for $\$ \mathrm{I} \_\mathrm{H}(\mathrm{t}) \$$ decreases; (ii) these noise intensities for three subgroups have the influence on the skewness and kurtosis of the stationary distribution for $\$ \mathrm{I}_{-} \mathrm{H}(\mathrm{t}) \$$, where the effect of the noise intensity for humans $\$ \backslash$ sigma_H $\$$ on the alteration of the distribution shape for $\$ \mathrm{I} \_\mathrm{H}(\mathrm{t}) \$$ is obvious, from skyscraping to pyknic and gradually migrating towards left as $\$ \backslash$ sigma_H $\$$ increasing.
\end{abstract}

\section{Hosted file}

The effect of stochastic environmental.pdf available at https://authorea.com/users/343835/ articles/470425-the-effect-of-stochastically-environmental-variability-on-transmissiondynamics-of-echinococcosis 